\title{
SINOPSE DE PSEUDOGNAPHALIUM KIRP. (ASTERACEAE-GNAPHALIEAE) NO BRASIL ${ }^{1}$ \\ LEONARDO PAZ DEBLE ${ }^{2}$ JOSÉ NEWTON CARDOSO MARCHIORI ${ }^{3}$
}

\section{RESUMO}

Predominantemente neotropical, o gênero Pseudognaphalium Kirp. é reconhecido como independente de Gnaphalium L. pela maioria dos autores atuais e inclui aproximadamente 95 espécies de ervas anuais, bianuais ou perenes. No Brasil, está representado por apenas cinco espécies: P. cheiranthifolium (Lam.) Hilliard \& Burtt, P. gaudichaudianum (DC.) A. Anderb., P. leucopeplum (Cabrera) A. Anderb., P. luteo-album (L.) Hilliard \& Burtt e P. mendocinum (Phil.) Deble \& Marchiori. Neste trabalho são propostas três novas sinonímias para $P$. cheiranthifolium (Gnaphalium erectum Vellozo, Gnaphalium riedelianum F. W. Klatt, Gnaphalium cheiranthifolium Lam. var. riedelianum (F. W. Klatt) Baker) e duas para Pseudognaphalium gaudichaudianum (Gnaphalium regnelli Sch.-Bip., Pseudognaphalium austrobrasilicum Deble \& Marchiori). Gnaphalium mendocinum Phil. é reabilitado da sinonímia de $P$. gaudichaudianum, sendo proposta uma nova combinação: Pseudognaphalium mendocinum (Phil.) Deble \& Marchiori. Um lectótipo para Gnaphalium erectum Vellozo é designado. São fornecidas chave dicotômica, sinonímias e distribuição geográfica das espécies.

Palavras-chave: Pseudognaphalium, Asteraceae, Gnaphalieae, Brasil.

\section{ABSTRACT}

Basically neotropical, the genus Pseudognaphalium is distinguished from Gnaphalium L. by most of the modern authors and includes 95 species of annual, biannual or perennial herbs. Only five species are native in Brazil: P. cheiranthifolium (Lam.) Hilliard \& Burtt, P. gaudichaudianum (DC.) A. Anderb., P. leucopeplum (Cabrera) A. Anderb., P. luteo-album (L.) Hilliard \& Burtt and P. mendocinum (Phil.) Deble \& Marchiori. This paper proposes three new synonymities to P. cheiranthifolium (Gnaphalium erectum Vellozo, Gnaphalium riedelianum F. W. Klatt, Gnaphalium cheiranthifolium Lam. var. riedelianum (F. W. Klatt) Baker), and two synonymities to Pseudognaphalium gaudichaudianum (Gnaphalium regnelli Sch.-Bip., Pseudognaphalium austrobrasilicum Deble \& Marchiori). Gnaphalium mendocinum Phil. is rehabilitated from of the synonymity of $P$. gaudichaudianum, being proposed a new combination: Pseudognaphalium mendocinum (Phil.) Deble \& Marchiori. A lectotype to Gnaphalium erectum Vell. is presently designated. A dichotomous key, the synonymity and the geographic distributions to all taxa are also furnished.

Key Words: Pseudognaphalium, Asteraceae, Gnaphalieae, Brazil.

\section{INTRODUÇÃO}

O gênero Pseudognaphalium Kirp. abriga cerca de 95 espécies de ervas anuais, bianuais ou perenes, providas de capítulos oblongos, campanulados ou hemisféricos, dispostos em corimbos ou panículas terminais, com brácteas involucrais com estereoma dividido e aquênios

ovados ou oblongos, de paredes normalmente papilosas, com pêlos mucilaginosos do tipo "twin-hairs".

Estreitamente relacionado à Achyrocline (Less.) DC., Pseudognaphalium difere por apresentar flores femininas em duas ou mais

1 Artigo recebido em 22/09/2006 e aceito para publicação em 08/11/2006.

2 Biólogo, Msc., bolsista da CAPES, doutorando do Programa de Pós-Graduação em Engenharia Florestal, Centro de Ciências Rurais, Universidade Federal de Santa Maria. CEP 97105-900, Santa Maria (RS). deble.biol@ gmail.com

3 Engenheiro Florestal, Dr., bolsista de Produtividade em Pesquisa CNPq, Professor Titular do Departamento de Ciências Florestais, Universidade Federal de Santa Maria. CEP 97105-900, Santa Maria (RS). balduinia@mail.ufsm.br 
séries e, principalmente, pelo tipo de pêlos nos aquênios. De Gnaphalium L., Pseudognaphalium afasta-se pela inflorescência em panículas ou corimbos terminais (versus pseudo- espigas folhosas), pelas brácteas involucrais monocromáticas com estereoma dividido (versus policromáticas com estereoma inteiro) e pelo pápus monomórfico (versus dimórfico).

\section{CHAVE PARA AS ESPÉCIES}

1a. Folhas com pêlos hirtos e glandulares na face adaxial, lanosas na abaxial

1b. Folhas lanosas ou lanuginosas em ambas as faces; pêlos glandulares, quando presentes, longos e entremesclados a pêlos lanosos

2a. Folhas discolores, densamente incano-lanosas na face abaxial. Capítulos de coloração estramínea até rufescente, com 3-4 mm de altura por 3-5 mm de diâmetro. Flores femininas, 160-270. Aquênios ovado-oblongos, de $0,4-0,5 \mathrm{~mm}$ 5. P. mendocinum (Phil.) Deble \& Marchiori

2b. Folhas suavemente discolores, lanosas a lanuginosas na face abaxial. Capítulos de coloração marfim, de 4,5-6 mm de altura por 5-7 mm de diâmetro. Flores femininas, 350-400. Aquênios oblongos, de 0,7 mm

\section{P. gaudichaudianum (DC.) A. Anderb.}

3a. Ervas anuais, herbáceas na base. Folhas não decurrentes ou breve-decurrentes (decurrência de até $4 \mathrm{~mm}$ ). Capítulos de 2,5-3,5 mm de altura. Flores femininas, 100-120. Aquênios oblongos, de 0,4-0,5 mm

4. P. luteo-album (L.) Hilliard \& Burtt

3b. Plantas sufrutescentes, lenhosas na base. Folhas decurrentes (decurrência de 8-20 mm). Capítulos de 4-6,5 mm de altura. Flores femininas, 130-250. Aquênios ovados ou ovado-oblongos, de 0,5-0,8 mm

4a. Folhas com longos pêlos glandulares entremesclados a pêlos lanosos. Capítulos de coloração amarelo-citrina ou dourada, de 4-5 mm de altura 1.P. cheiranthifolium (Lam.) Hilliard \& Burtt

4b. Folhas desprovidas de pêlos glandulares. Capítulos de coloração estramínea ou branco-amarelada, de 5-6,5 mm de altura 3. P. leucopeplum (Cabrera) A. Anderb.

\section{LISTA DE ESPÉCIES}

\section{Pseudognaphalium cheiranthifolium (Lam.) Hilliard \& Burtt}

O. M. Hilliard \& B. L. Burtt, Bot. Journ. of the Linn. Soc., 82, p. 205, 1981.

Basiônimo: Gnaphalium cheiranthifolium Lam. Encyclopédie Méthodique Botanique 2, p. 752, 1786.

TIPO: Uruguai, Montevideo, Commerson. Holotypus P n.v., Foto digitalizada do tipo P!

$=$ Gnaphalium erectum Vellozo, Fl. Flumin. Tomo VIII, t. 98, 1825 (29) [Tipo: Material original desconhecido. Lectotypus (aqui designado): tab. 98, in Vellozo, Fl. Flum. tomo VIII, 1829.]; syn. nov.

= Gnaphalium citrinum Hook. \& Arn., Bot. Beechey Voyage, p. 31, 1830 [Tipo: Chile, Hualqui, C. E. O. Kuntze s.n., 20.II.1892, Foto digitalizada do tipo NY!];

= Gnaphalium paniculatum Colla, Mem. Reale Accad. Sci. Torino 38, p. 17, tab. 26, 1835 [Tipo: Chile, Valparaíso, leg. Bertero 1033. Holotypus n.v. Isotypus ? RB!]

= Gnaphalium valdivianum Phil., Linnaea 29, p. 6, 1857;

= Gnaphalium araucanum Phil., Anales Univ. Chile 43, p. 502, 1873;
= Gnaphalium riedelianum $\mathrm{F}$. W. Klatt, Linnaea, v. 42, p. 115, 1878 [Tipo: Brasil, leg. Riedel, Holotypus n. v. Isotypus R!]; syn. nov.

$=$ Gnaphalium cheiranthifolium Lam. var. riedelianum $(\mathrm{F}$. W. Klatt) Baker, Mart., Fl. Bras. 6, 3, p. 122, 1882; syn. nov.

= Gnaphalium acutifolium Phil., Anales Univ. Chile 90, p. 12, 1895;

$=$ Gnaphalium cheiranthifolium Lam. var. citrinum (Hook. \& Arn.) Kuntze, Rev. Gen. Pl. 3, p. 151, 1898;

$=$ Gnaphalium cheiranthifolium var. paniculatum (Colla) Skottsb., Kongl. Svenska Vetensk. Acad. Handl. 51, n. 9 , p.5, 1914.

Distribuição \& Habitat: Desde o Chile, Argentina e Bolívia, até o Uruguai e o sul, sudeste e nordeste do Brasil. Habita regiões campestres e beira de matas subtropicais, desde o nível do mar até cerca de 2.500 m.s.m. Floresce e frutifica com maior intensidade no final do verão e durante o outono; material fértil, todavia, pode ser encontrado ao longo de todo ano. 


\section{Pseudognaphalium gaudichaudianum} (DC.) A. Anderb.

A. Anderberg, Opera Bot. 104, p. 147, 1991.

Basiônimo: Gnaphalium gaudichaudianum DC., Prodromus 6, p. 226, 1837.

TIPO: Brasil, Rio de Janeiro, C. Gaudichaud-Beaupré 673. Holotypus G-DC n.v., foto digitalizada do holótipo G-DC! Isotypus R!

= Gnaphalium gaudichaudianum DC. var. subrufescens DC., Prodromus 6, p. 226, 1837 [Tipo: Brasil, prov. Rio Grande, F. Sellow 983. Holotypus G-DC n.v. Isotypus R!]; = Gnaphalium cheiranthifolium Lam. var. subrufescens (DC.) Baker, Mart., Fl. Bras. 6, 3, p. 122, 1882;

$=$ Gnaphalium cheiranthifolium Lam. var. gaudichaudianum (DC.) Baker, Mart., Fl. Bras. 6, 3, p. 122, 1882;

= Gnaphalium regnelli Sch.-Bip., nom. nud. [Tipo: Brazil, prov. Minas Gerais, Regnell I-272. Holotypus n.v. Isotypus R!] syn. nov.;

= Pseudognaphalium austrobrasilicum Deble \& Marchiori, Balduinia, n. 6, p. 4, 2006. [Tipo: Brasil, Rio Grande do Sul, São Sepé, BR 392, p. Santa Maria, após o viaduto da BR 290 , em campo, na beira da estrada, abundante, erva glandulosa, ereta, de $90 \mathrm{~cm}$, capítulos brancos, odor fétido intenso, L. P. Deble \& A. S. de Oliveira 5.000, 02.X.2004. Holotypus SI!] syn. nov.

Distribuição \& Habitat: Ocorre no sul e sudeste do Brasil, bem como no nordeste da Argentina. Habita regiões campestres, desde o nível do mar até os 2.000 m.s.m. Floresce e frutifica na primavera.

\section{Pseudognaphalium leucopeplum (Cabre-} ra) A. Anderb.

A. Anderberg, Op. Bot 104, p. 147, 1991.

Basiônimo: Gnaphalium leucopeplum Cabrera, Bol. Soc. Argent. Bot. 9, p. 383, 1961.

TIPO: ARGENTINA, Prov. Buenos Aires, Juancho, en dunas semifijas, A. L. Cabrera 2.686, 11.I.1933. Holotypus LP!

Distribuição \& Habitat: Ocorre na Argentina, Uruguai e Rio Grande do Sul. Habita solos arenosos, principalmente em dunas litorâneas. Floresce e frutifica no verão-outono.
4. Pseudognaphalium luteo-album (L.) Hilliard \& Burtt

O. M. Hilliard \& B. L. Burtt, Bot. Journ. of the Linn. Soc., 82, p. 206, 1981.

Basiônimo: Gnaphalium luteo-album L., Species Plantarum, p. $851,1753$.

TIPO: EUROPA, sine loco. Holotypus LINN 900 n.v., foto digitalizada do holótipo LINN!

Distribuição \& Habitat: Espécie de regiões subtropicais e temperadas, de quase todo planeta; no Brasil, sua ocorrência é duvidosa, tendo sido analisado um único exemplar, coletado por Sellow, com etiqueta indicando "in Brasilia, prov. Rio Grande”. Esta espécie ocorre no Uruguai, em regiões próximas a divisa com o Brasil, motivo pelo qual, $P$. luteo-album foi incluída nesta sinopse. Floresce e frutifica no final do inverno e primavera.

\section{Pseudognaphalium mendocinum (Phil.) Deble \& Marchiori, comb. nov. \\ Basiônimo: Gnaphalium mendocinum Phil., Anales Univ. Chile 36, p. 184, 1870.}

TIPO: ARGENTINA, Mendoza, R. A. Philippi, s.n. Holotypus SGO n.v. Isotypus CORD!

Distribuição \& Habitat: Habita campos arenosos, desde o Chile, Argentina e Uruguai, até o sul e sudeste do Brasil. Floresce e frutifica principalmente no verão-outono.

\section{BIBLIOGRAFIA}

Anderberg, A. Taxonomy and phylogeny of the tribe Gnaphalieae (Asteraceae). Opera Bot., Copenhagen, n. 104, p. 1-195, 1991.

Baker, J. F. Compositae. In: Martius, C. F. Flora Brasiliensis. München, Wien and Leipzig, 1882, v. 6, n. 3, p.1-132.

Cabrera, A. L. Observaciones sobre las InuleaeGnaphalineae (Compositae) de América del Sur. Bol. Soc. Argent. Bot., La Plata, v. 9, p. 362-383, 1961. 
Cabrera, A. L. Compositae. In: Cabrera, A. L. Flora de la Provincia de Buenos Aires (Argentina). Buenos Aires: INTA, 1963. Tomo IV, parte VI, p. 161-166.

Cabrera, A. L. Compositae. In: Correa, M. N. Flora Patagonica (Argentina). Buenos Aires: INTA, 1971. Tomo VIII, parte VII, p. 109-115.

Cabrera, A. L. Compositae. In: Burkart, A. Flora Ilustrada de Entre Ríos (Argentina). Buenos Aires: INTA, 1974. Tomo VI, parte VI, p. 315-317.

Cabrera, A. L. Compositae. In: Cabrera, A. L. Flora de la Provincia de Jujuy (Argentina). Buenos Aires: INTA, 1978. Tomo VIII, parte X, p. 275-288.
Deble, L. P., Marchiori, J. N. C. O gênero Pseudognaphalium Kirp. (Asteraceae-Gnaphalieae) no sul do Brasil. Balduinia, Santa Maria, n. 6, p. 1-13, 2006.

De Candolle, A. P. Prodr. Syst. Nat. Reg. Veget. Paris, 1837. v. 6, p. $218-268$.

Freire, S. E. Asteraceae. Tribu IV. Inuleae. Flora Fanerogámica Argentina, Córdoba, n. 14, p. 1-60, 1995.

Hilliard, O. M., Burtt, B. L. Some generic concepts in Compositae-Gnaphaliinae. Bot. J. Linn. Soc., n. 82, p. 181-232, 1981.

Klatt, F. W. Pflanzenkundae. Linnaea, Berlin, v. 42, p. 112-113, 1878. 